\title{
Application of the Four Colour Theorem to Identify Spatial Regional Poles and Turnpikes of Economic Growth
}

\author{
A. JAKIMOWICZ ${ }^{a, *}$ AND D. RZECZKOWSKI ${ }^{b}$ \\ ${ }^{a}$ Institute of Economics, Polish Academy of Sciences, Palace of Culture and Science, \\ Pl. Defilad 1, PL-00901 Warsaw, Poland \\ ${ }^{b}$ Department of Quantitative Methods, Faculty of Economic Sciences, University of Warmia and Mazury in Olsztyn, \\ M. Oczapowskiego 4, PL-10719 Olsztyn, Poland
}

\begin{abstract}
This paper presents a method for identifying regional poles and the turnpikes of growth based on the following foundations: four colour theorem, Wikinomics business model in the form of platforms for participation, evaluation of the functionality of websites run by public administration municipal offices, and dual graph reduction. The province of Warmia and Mazury, which is the subject of the study, is one of the poorest provinces in Poland in terms of economic development. It is therefore natural that the growth of this region requires external enterprise sources. This role can be best performed primarily by websites run by municipal offices, which initiate business activity in their corresponding areas, and consequently, can be regarded as Wikinomics platforms of participation. Using the $k$ means clustering method, these websites were divided into four separate quality classes. These classes were assigned four various colours, which were subsequently used for preparing the map of the province. Each municipality was marked with a colour corresponding to the quality class of the website run by the state administration unit operating in a given area. The system of colours resulting from the four colour theorem and a corresponding dual graph serve as a frame of reference with regard to each empirical colour distribution and to another, related, dual graph. Thus, the four colour theorem describes the largest diversity of regional growth poles. The measure of the economic growth of the region is a degree of reduction of the dual graph corresponding to the empirical colour distribution, which identifies actual growth poles and determines the turnpikes of growth. The ultimate development objective, although not always achievable, is a reduction of the dual graph to a single vertex, when all municipal offices in the province have websites of the highest quality.
\end{abstract}

DOI: 10.12693/APhysPolA.133.1362

PACS/topics: 89.65.Gh, 02.10.Ox

\section{Introduction}

The relations between technology and economic development, emphasized by almost all schools of macroeconomics, have been known for a long time. This even led to defining the term of "peak technology", denoting the technology dominating in a given period, with teleinformatics fulfilling this role at present. Although until recently it was investigated as the factor behind growth and competitiveness mainly in the private sector, it has now found multiple applications in the public sector. Comprehensive aspects of those changes are described by a new school in economics - Wikinomics.

Teleinformatics in public administration sector contributes to the creation of appropriate conditions in which two basic groups of business entities, i.e. enterprises and households, operate. This takes effect primarily by improving the relationship between this sector and its economic surrounding, by streamlining the process of providing already existing services and implementing new ones and by triggering the development of local industry and trade. What is of great importance at this point is knowledge sharing, which takes place through local government websites. Therefore, in Wikinomics terms, they

${ }^{*}$ corresponding author are referred to as platforms of participation. Website development provides evidence that the economic growth pole is emerging in a given community or region, since those websites become the main channels of economic information flow.

According to the research hypothesis assumed in the paper, the process describing the emergence of municipal growth poles and their merging within the province into turnpikes of growth can be identified with the use of the four colour theorem. Consequently, economic development can be described in graph theory terms and it consists in reduction of the dual graph corresponding to the map of the province of Warmia and Masuria, with quality classes of the local government websites plotted on the map. This will help to reveal not only the mere fact of local economic development or regress, but also its qualitative and quantitative characteristics. As a result of applying the above described method, it was demonstrated that achievements of past generations provide the basic source of the current economic growth in the province under examination. This proves the need to introduce an urgent change in the state policy with regard to initiating economic growth and development in this region.

Identification of spatial regional poles and turnpikes of economic growth is a challenge which has not yet been properly addressed [1]. However, it may be tackled in a 
non-standard manner by applying the four colour theorem. The subject of study is the region of Warmia and Masuria which is one of the least developed voivodships of Poland in terms of economy. The method applied involves a Wikinomics business model which entails platforms for participation. The role of such platforms is played by websites of the administrative authorities of municipalities. An analysis of the functionality of those websites provided information on the economic growth of the municipalities. Subsequently, by using the $k$-means clustering method, four classes of functionality of platforms for participation of the municipalities were defined. Each municipality was marked on the map of WarmianMasurian voivodship in a colour that reflects the functionality of its website. In this way, spatial regional poles and turnpikes of economic growth were identified. This resulted in a dual graph presenting the economic growth of the voivodship. To analyse the graph, a frame of reference defined by another dual graph which complies with the four colour theorem and shows the entire lack of poles of growth, was used.

\section{The four colour theorem}

The four colour theorem reads as follows: regions on every planar map can be coloured in only four colours in such a manner that each two adjacent regions have different colours [2-3]. Adjacent regions mean regions which border each other along a line, not in a particular point or even in a finite number of points. In addition, a region means a cohesive area consisting of a single area because otherwise it would be easy to name an example of a fiveregion map, where each region would border the other four regions. In other words, the four colour theorem is about normal maps that meet two requirements: they do not border more than three regions in one point and no region fully encircles any other region.

Every non-normal map can be paired with a normal map that requires at least the same number of colours. Thus, since the four colour theorem is valid for normal maps, it is valid for all maps. Every normal planar map complies with the following equation:

$$
\begin{gathered}
4 p_{2}+3 p_{3}+2 p_{4}+p_{5}-p_{7}-2 p_{8} \\
-3 p_{9}-\cdots-(N-6) p_{N}=12,
\end{gathered}
$$

where $p_{n}$ is the number of regions on the map which have exactly $n$ neighbours, and $N$ is the largest number of neighbours of any region; $n=0$ and $n=1$ are excluded since normal maps do not have enclaves or islands. Therefore, the equation has to begin with $p_{2}$. This way, every $p_{n}$ is either positive or equals zero and it is positive only if $n<6$. This means that at least one number out of $p_{2}, p_{3}, p_{4}$, or $p_{5}$ has to be positive as the sum on the left side of Eq. (1) should be positive to equalise a positive number on the right side. One of the regions has to have two, three, four, or five neighbours. There does not exist a normal planar map where each region has six or more neighbours.
The foregoing allows two concepts underlying the graph theory to be defined: an unavoidable set and reducible configurations. A set is unavoidable if it contains a group of configurations that consist of a region with two neighbours, a region with three neighbours, a region with four neighbours and a region with five neighbours. Its name is derived from the fact that every normal map has to include at least one of the four configurations referred to above. A configuration is reducible if it cannot appear on a minimal five-chromatic normal map, i.e. on the smallest map that requires five colours. The discovery of an unavoidable set of reducible configurations is sufficient to prove the four colour theorem.

The four colour theorem was formulated in 1852 by Francis Guthrie, a mathematics student at the University College of London. When colouring a map of England, he noticed that only four colours are needed to make every two adjacent counties have different colours. Mathematicians found it very difficult to prove the four colour theorem, despite its simplicity, and it took them 125 years to prove it. The proof of concept was announced in 1976 by Appel and Haken who based the theorem on the structure of an unavoidable set of 1,936 reducible configurations (as it turned out later, ultimately there were 1,482 configurations) [4]. The proof was achieved with computer calculations. In subsequent years, that proof was improved several times, but the need to use a computer was not eliminated [5-9]. This means that verification of the proof requires quite a different understanding than the one applied earlier in mathematics. This understanding cannot be verified by the human mind. It sparked a discussion about the nature of a mathematical proof [10]. Certain philosophical repercussions of the four colour theorem were also debated by indicating that it is not an a priori truth, which would be required in traditional mathematics, but an a posteriori truth [11, 12]. Perhaps a traditional proof of the four colour theorem exists and will be discovered in the future.

\section{Wikinomics and platforms for participation}

Wikinomics is a new school of economics that focuses on non-standard business models established in the digital economy era [13]. One of these models are platforms for participation which involve products and technical infrastructure shared with peer communities to let them use such products and infrastructure to define new values and trigger innovative undertakings. Platforms for participation are based on all of the four Wikinomics principles: openness, peering, sharing and acting globally. Such a business model allows companies to act on a wider stage - when compared to the traditional model - where many various partners can operate their own economic activities or improve existing solutions. This results in an increase in the productive capacity of companies without additional fixed costs to be incurred by them. A website with an e-commerce system for warehousing, purchasing, and distributing goods could 
be an example of a platform for participation. They are very frequently used to establish on-the-fly partnerships with software developers who add new value to what has already been achieved. Companies sharing their software services and databases via the application programming interface (API) contribute to generating fluid webs of business partnerships, dubbed developer ecosystems. Establishment of open platforms for participation increases the speed, coverage and chances of an innovation's success.

One of the most essential types of platforms for participation are web service mashups. They are websites that combine various ready-to-go services or applications made available via other websites, thus resulting in a new facility of higher quality and functionality. In other words, it is about remixing data and software into recombinant creations. This phenomenon is increasingly popular since more and more companies are sharing their APIs.

Platforms for participation can focus on various products or services, virtually anything that runs software, and that makes them useful in the public sector, especially in public administration. Presently, websites of the administrative authorities of municipalities are far behind platforms for commerce. This means that the largest sources of public information are, in fact, not used, though they could be the basis for many new public services. Therefore, society exploits the most important factors of regional economic growth to a rather limited extent. There is an urgent need to develop platforms for participation in public administration so that citizens and companies communicate freely with their government and the rest of the networked world. Mashups in public administration are an opportunity to extract new value from public information and to use it in business better. A good example of this is the integration of public information bulletins with other non-proprietary data sources. Websites of public administration authorities may be platforms for grassroots action and may include platforms for public disclosure and platforms for neighbourhood knowledge.

\section{Assessment of the functionality of the websites of the municipal administrative authorities}

An assessment of the functionality of the websites of municipal administrative authorities was carried out in accordance with sixteen criteria represented by symbols from A01 to A16 [14]. The binary method was applied which entails giving a website a point if a given criterion is met or a zero when it is not met. Subsequently, points were summed up. Thus, the functionality index of a website was a number from a closed interval $[0,16]$. Using the $k$-means method, the websites were divided into homogeneous subsets. This led to natural separation of four subsets reflecting four Wikinomics resource categories of the websites in question: low-quality, av- erage quality, high quality, and very high quality. The study was carried out twice - in 2009 and 2012.

Table I characterises four clusters representing the Wikinomics categories of quality of the websites of the municipal administrative authorities. It also features percentage absorption of the website functionality criteria in individual years and clusters. The results shown in Table I are presented spatially, as two maps of the Warmian-Masurian voivodship, in which each municipality is given a colour corresponding to the category of the quality of its website in a given year.

\section{Specification of the frame of reference in the study}

The four colour theorem is - despite appearances - of little significance for map makers and cartographers [3]. In fact, science lacks profitable applications of the theorem, i.e. applications solving an important scientific problem. To date, there have been only a few attempts to apply the four colour theorem to various fields of science and all of them were rather unsuccessful [15-20]. This paper seeks to demonstrate that this theorem is of great importance not only in the identification of economic growth poles, but also in regional growth measurement.

The four colour theorem was used to establish a reference frame for the study. To this end, a map of municipalities of the Warmian-Masurian voivodship was coloured in four colours, as per the theorem. The map is presented in Fig. 1. It allowed a corresponding dual graph to be created. The capital cities or towns of all the municipalities were marked on the map and then the capital cities or towns of neighbouring municipalities were linked with each other by roads crossing their borders. The result is a dual graph of the original map and it is also presented in Fig. 1. Here, the capital cities or towns of the municipalities are the vertices of the graph and the roads are its edges. The dual graph was drawn in such a way that all edges are straight line sections. The edges of the graph divide the plane into faces. The capital cities or towns of the municipalities, i.e. the vertices, correspond on the original map to the faces of the dual graph. After eliminating instances of full encirclement of one municipality by another, the map of the voivodship is a normal map. This means that exactly three edges converge at each vertex of the dual graph, hence all the faces have to be triangles. Such a dual graph is called a triangulation. A number of edges converging at a given vertex is called the degree of the vertex and is equal on the original map to the number of neighbours of the municipality represented by that vertex.

According to the terms and definitions used in dual graphs, a configuration is part of a triangulation consisting of a set of vertices and edges that connect them. The contour of a graph is a finite path consisting of vertices and edges which begins and ends in the same vertex, which does not cross itself and which divides the graph into two parts: internal and external. An external con- 
tour is called the boundary ring of the dual configuration. On the original map of the voivodship in question (Fig. 1), there are thirty-nine external municipalities encircling their centre. The municipality of Dubeninki was omitted for obvious reasons. After the dual graph was created, they were replaced with the boundary ring of the dual configuration, i.e. with an external contour consisting of thirty-nine vertices and thirty-nine edges. The dual configuration shown in Fig. 1 is dubbed a thirtynine-ring configuration as its ring has thirty-nine vertices. It corresponds to a ring of thirty-nine municipalities that encircle the original configuration.

TABLE I

Four clusters representing the Wikinomics categories of quality of the websites of the municipal administrative authorities.

\begin{tabular}{c|c|c|c|c|c|c|c|c}
\hline \hline Years & \multicolumn{9}{|c|}{2009} & \multicolumn{4}{c}{2012} \\
\hline Clusters & $\begin{array}{c}\text { Low } \\
\text { quality }\end{array}$ & $\begin{array}{c}\text { Average } \\
\text { quality }\end{array}$ & $\begin{array}{c}\text { High } \\
\text { quality }\end{array}$ & $\begin{array}{c}\text { Very } \\
\text { high } \\
\text { quality }\end{array}$ & $\begin{array}{c}\text { Low } \\
\text { quality }\end{array}$ & $\begin{array}{c}\text { Average } \\
\text { quality }\end{array}$ & $\begin{array}{c}\text { High } \\
\text { quality }\end{array}$ & $\begin{array}{c}\text { Very } \\
\text { high } \\
\text { quality }\end{array}$ \\
\hline points (min-max) & 0 & $5-9$ & $10-11$ & $12-16$ & 0 & $7-10$ & $11-13$ & $14-16$ \\
\hline centroid & 0 & 7.72 & 10.45 & 13.35 & 0 & 9 & 11.75 & 14.45 \\
\hline $\begin{array}{c}\text { number } \\
\text { of municipalities }\end{array}$ & 17 & 22 & 37 & 40 & 6 & 11 & 49 & 50 \\
\hline
\end{tabular}

Encoded functionality criteria of the websites of the municipal administrative authorities and their absorption in individual years and clusters (per cent)

A01
A02
A03
A04
A05
A06
A07
A08
A09
A10
A11
A12
A13
A14
A15
A16

A01

$\mathrm{A} 03$

A04

A05

A06

$\mathrm{A} 07$

A09

A10

A11

A12

A14

A 15

\begin{tabular}{|c|c|c|}
\hline 0 & 100 & 100 \\
0 & 100 & 100 \\
0 & 0 & 3 \\
0 & 55 & 92 \\
0 & 77 & 100 \\
0 & 32 & 57 \\
0 & 68 & 92 \\
0 & 5 & 38 \\
0 & 14 & 27 \\
0 & 14 & 54 \\
0 & 73 & 92 \\
0 & 77 & 97 \\
0 & 73 & 95 \\
0 & 86 & 97 \\
0 & 0 & 0 \\
0 & 0 & 5
\end{tabular}

\section{Identification of economic growth poles as a method to reduce a dual graph}

Regional poles of economic growth should be understood as drivers which drive innovation and contribute to the improvement of economic performance of companies and their surrounding industries [21-22]. In terms of Wikinomics, a growth pole is present wherever advanced ICT tools are used in business. It may be a website of the administrative authorities of a municipality which serves as a platform for participation and integrates the economic forces of a given municipality. Websites of very high quality have the best chance to become local growth poles. If there are municipalities in a given voivodship with such websites, it is very probable that there are growth poles. In addition, the spatial distribution of such municipalities may indicate economic growth in space and pinpoint obstacles.

Two frames of reference can be used to assess the economic growth of the region of Warmia and Masuria.The

\begin{tabular}{c|c|c|c|c}
100 & 0 & 100 & 100 & 100 \\
100 & 0 & 73 & 100 & 100 \\
10 & 0 & 0 & 2 & 76 \\
100 & 0 & 27 & 98 & 100 \\
100 & 0 & 100 & 100 & 100 \\
98 & 0 & 55 & 96 & 98 \\
100 & 0 & 82 & 94 & 100 \\
93 & 0 & 9 & 24 & 94 \\
73 & 0 & 18 & 59 & 100 \\
95 & 0 & 100 & 100 & 100 \\
100 & 0 & 55 & 78 & 100 \\
100 & 0 & 100 & 100 & 100 \\
100 & 0 & 100 & 100 & 100 \\
100 & 0 & 100 & 98 & 100 \\
48 & 0 & 0 & 20 & 46 \\
20 & 0 & 0 & 4 & 34
\end{tabular}

first of them is the map of the voivodship in question presented in Fig. 1, coloured in accordance with the four colour theorem. The dual graph created on its basis corresponds to an instance of an absolute lack of growth poles. The second of them applies when all the municipalities have websites of the highest quality. In such a case, a dual graph is reduced to one vertex, which means that the entire voivodship has become a large growth pole and has a dominant position in the national economy. Naturally, those two frames of reference are quite unlikely to exist in the real world. Thus, the reality must be in between. However, they point to the nature of the method of identification of growth poles and turnpikes. It consists in reducing dual graphs corresponding to real maps (when compared to a graph corresponding to a master map). Such a reduction is done by combining municipalities into clusters that correspond to individual categories of the quality of their websites.

Figure 2 shows a map of the municipalities of the Warmian-Masurian voivodship divided into the cate- 
gories of the quality of the websites of the municipal public administrative authorities in 2009. The details are presented in Table I. Its comparison to the master map (Fig. 1), where no growth poles are present, shows essential information on regional economic growth. The dual graph corresponding to the map in Fig. 2 has far fewer vertices and edges than the dual graph created based on the master map. The graph created based on the map of 2009 shows a certain characteristic of the voivodship in question, i.e. the dominance of historically conditioned infrastructural growth poles in that voivodship. Historical conditions result from the fact that a significant part of the Warmian-Masurian voivodship was once part of East Prussia and it was finally regained by Poland in 1945 as part of the Regained Territories. Currently, it borders the Kaliningrad Oblast of Russia in the north. Poland took over these areas with their existing roads, railway tracks and inland waterways.

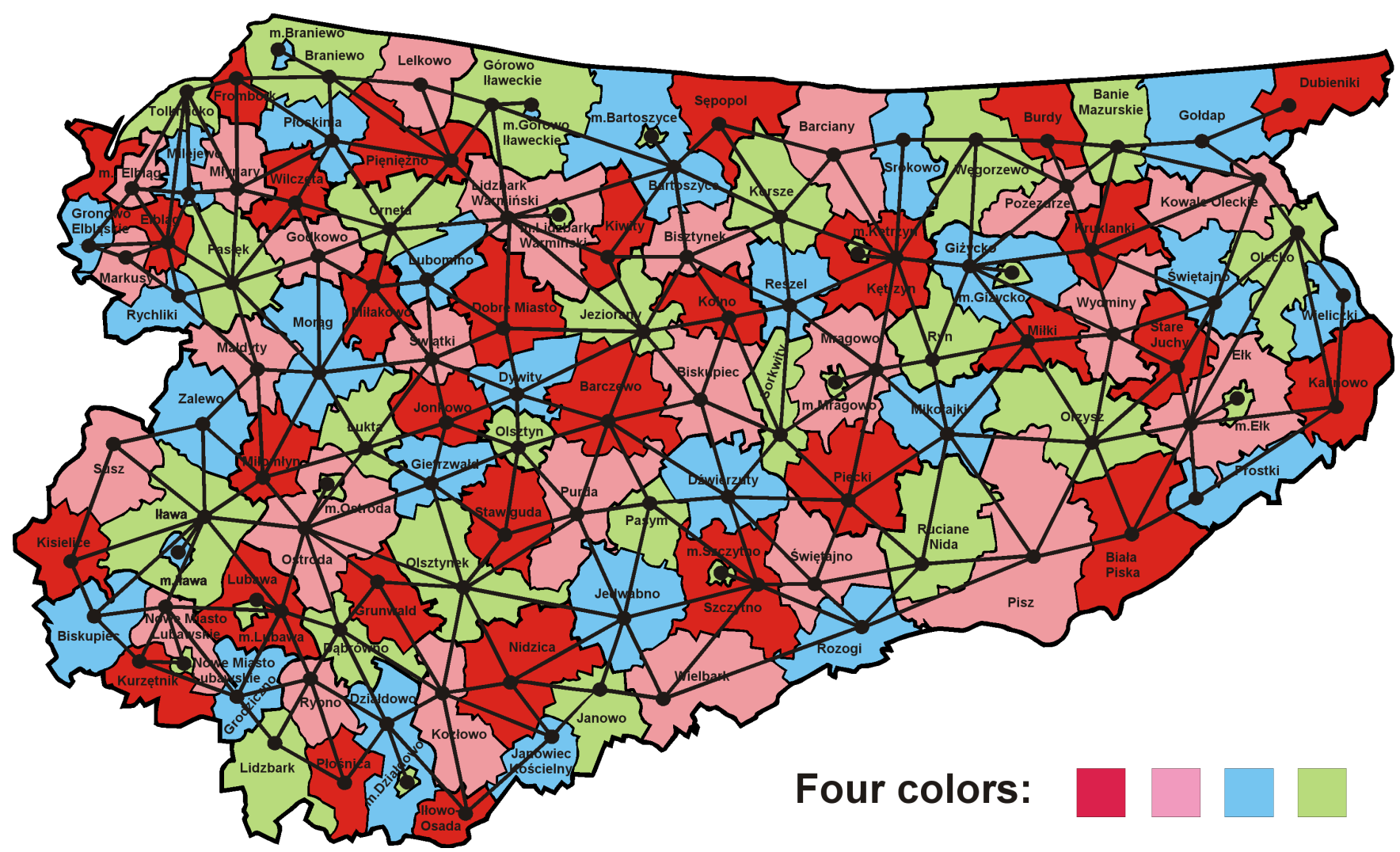

Fig. 1. Map of the municipalities of the Warmian-Masurian voivodship coloured in accordance with the four colour theorem and a corresponding dual graph. It is a frame of reference without growth poles.

The municipal websites of high and very high quality are strongly connected with transportation infrastructure erected before 1945 . Western areas of the voivodship include a cluster of websites of very high quality situated along the north-south axis. In those municipalities, there is the Elblag Canal built in the 19th century. Moreover, there are the docks in Elbląg overseeing the traffic of cargo barges on the Vistula Lagoon between Poland and Russia, as well as the roads between Nidzica, Olsztynek, Ostróda, Elbląg, and Królewiec with two road border crossings: Gronowo-Mamonowo and Grzechotki-Mamonowo II. Finally, there is the railway line between Malbork, Elbląg, Braniewo, Królewiec with the rail border crossing Braniewo-Mamonowo. The second cluster of websites of the highest quality is located to the east, also along the north-south axis, which partially results from geographical conditions. Two roads are situated there. The first of them (Road No. 63) goes through Pisz, Orzysz, Giżycko, and Węgorzewo, and before 1945 it led to today's Kryłowo on the Russian side of the border (there is no border crossing there now). The second of them (Road No. 65) goes through Ełk, Olecko, Gołdap, and ends with the Polish and Russian road border crossing at Gołdap-Gusev. On the map shown in Fig. 2, there is also a zone of municipalities located in the centre of the voivodship, along the south-west and north-east axis. National Road No. 16 (Iława-Ostróda-Olsztyn-MrągowoOrzysz-Ełk) and a railway line (Iława-Ostróda-OlsztynCzerwonka-Korsze) go through there, ending with a rail border crossing Skandawa-Zheleznodorozhny. Slightly to the left from the centre of the map, there is a barely visible growth line Olsztynek-Olsztyn-Dobre MiastoLidzbark Warmiński-Bartoszyce connected with National Road No. 51 and ending with the road border crossing Bezledy-Bagrationovsk. 
A comparison of the dual graphs in Figs. 1 and 2 proves that exerting an impact by local growth poles on the surroundings entails reducing the number of vertices and edges of the graph corresponding to the actual map. Edges should be interpreted as obstacles to growth occurring among neighbouring regions. The reasons for such phenomena vary and each of them needs to be analysed individually. Figure 2 shows that the largest numbers of obstacles are in the north and the south of the voivodship.

Figure 3 presents, as per Table I, the location of the Wikinomics growth poles in 2012. The dual graph corresponding to that map has far fewer edges than the dual graph of 2009, which means that new growth poles have appeared and many obstacles have been eliminated. The remaining obstacles are present in the north of the voivodship; therefore, investment activities and innovation should be intensified there.

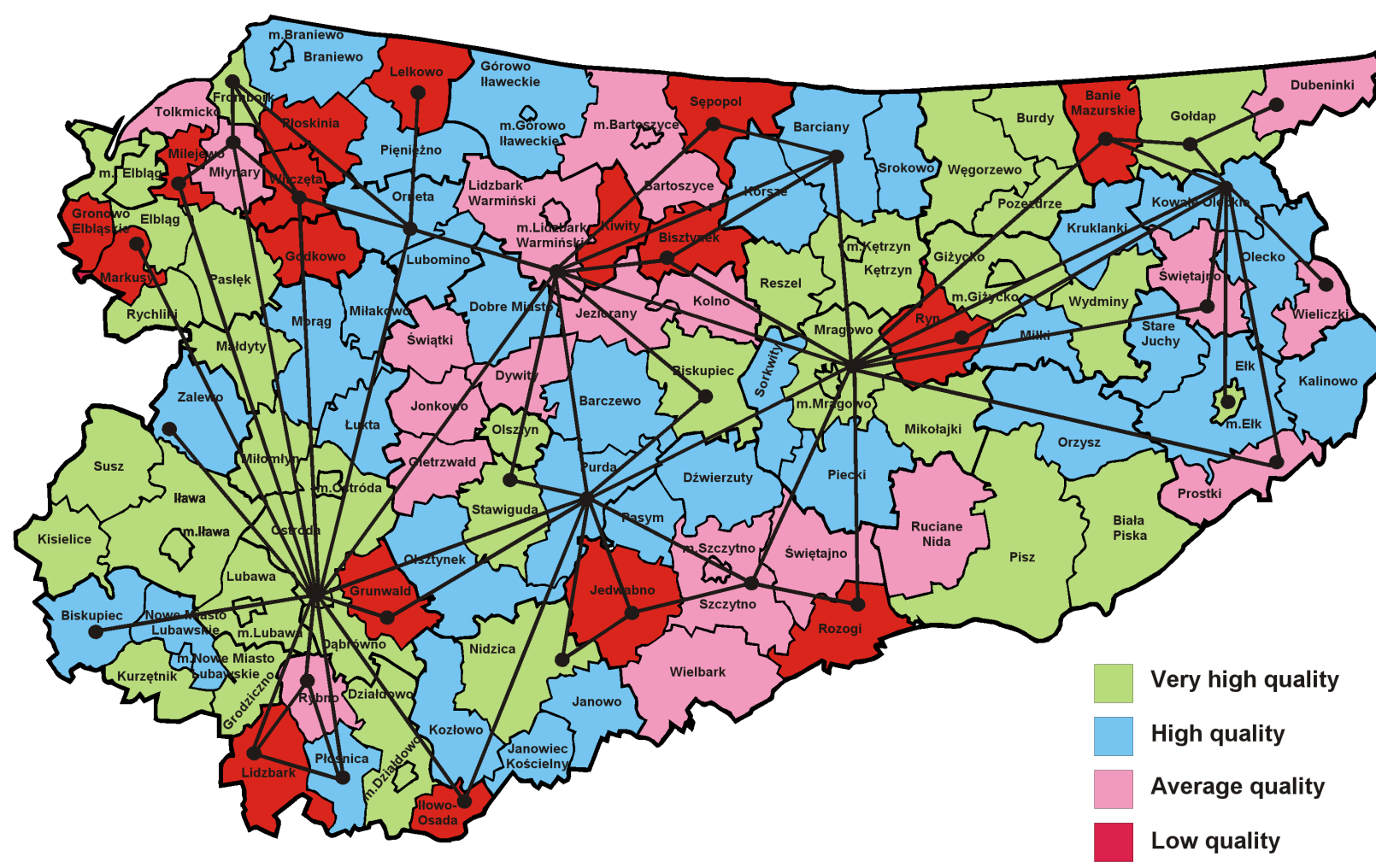

Fig. 2. Map of the municipalities of the Warmian-Masurian voivodship divided into categories of the quality of the websites of municipal public administrative authorities in 2009 and a corresponding dual graph.

What notably contributed to such rapid development was the construction of a $2,292 \mathrm{~km}$ long fibre-optic network with adequate infrastructure which allowed residents, companies, and state authorities in that voivodship to use broadband Internet. This investment was realised in 2008-2015 and its value amounted to PLN 327,041,042.07 (EUR 78,250,715.91) [23, 24]. This type of economic growth can be associated with the turnpike theory in economics $[25,26]$. The idea originates from an American English word that means a highway. The problem concerns setting an economy on the path of maximal balanced growth, as defined by von Neumann, which corresponds to the use of the peak technology to generate the highest revenues. The solution to this dilemma is similar to the choice between the use of a turnpike and a network of minor roads when one wants to arrive at one's destination as quickly as possible. If the starting point and the destination are at a great distance from each other, it is always better to use a turnpike, even if one needs to incur costs at intermediate stages. Such an understanding was applied to the voivodship in question since according to Wikinomics ICT is currently a peak technology, thus the lines connecting regional growth poles have to be called turnpikes.

\section{Methodological implications}

Identification of regional growth poles poses a great challenge for authorities in every voivodship. This aim can be reached by various methods developed by economists, as comprehensively described by Girejko [1] The methodology he presented concerns sustainable regional development in Belarus, therefore it may seem useful for examining the Warmian-Masurian voivodship in view of certain similarities between those regions. However, the majority of the proposed methods are of the qualitative type, and they often refer to expert evalua- 
tions, which can make their objectivity a questionable issue. Difficulties also lie in the need to determine a reliable frame of reference to be used for evaluation of economic achievements of all communes making up a given voivod- ship. Another frequently overlooked aspect includes spatial conditions for conducting business activities, resulting from the geographical location. Such objects as lakes, forests, or rivers can be both growth factors and barriers.

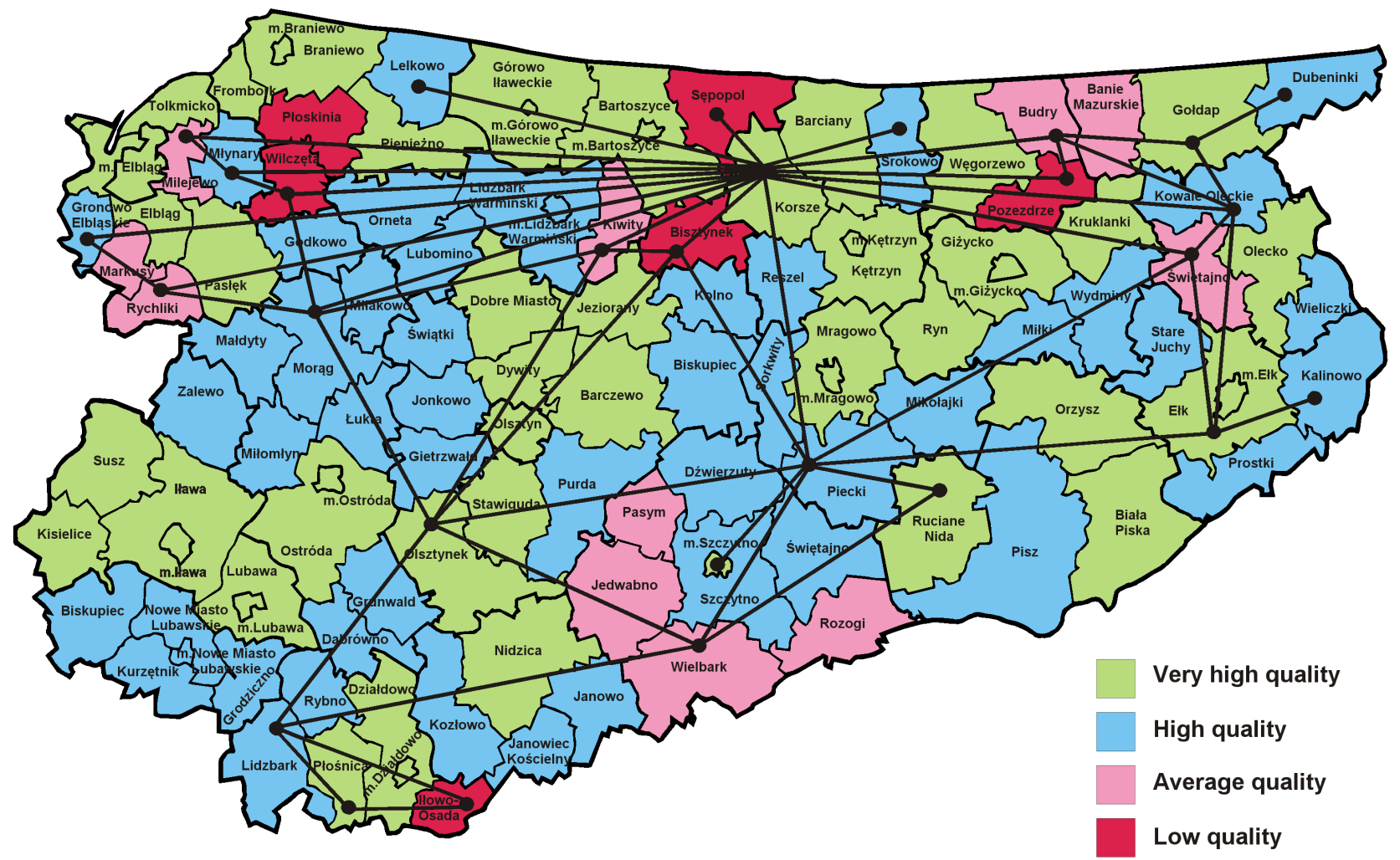

Fig. 3. Map of the municipalities of the Warmian-Masurian voivodship divided into the categories of the quality of the websites of municipal public administrative authorities in 2012 and a corresponding dual graph.

The application of the four colour theorem provides significant progress in studies concerning regional poles and turnpikes of economic growth, since it removes the majority of cognitive constraints of the classical methods. In comparison to the conventional approach applied so far in economy, the benefits of the methodology presented in the paper are as follows:

1. The four colour theorem establishes two natural frames of reference for evaluation of each empirical distribution of poles and turnpikes of economic growth in the communes. The first of them consists in the possibility of referring any empirical map of the examined voivodship to the map presenting colour distributions consistent with the four colour theorem. This permits to evaluate the reduction degree for the dual graph corresponding to the empirical map, which is tantamount to eliminating a specific number of growth barriers. Consequently, identification of growth poles is made possible. The second frame of reference is formed when the dual graph of the empirical map is reduced to a single vertex and the entire voivodship becomes a growth pole for the national economy. The entire reality lies between those two hypothetical states.
2. The method is based on ICT, which is the current peak technology, and combines many qualitative and quantitative factors affecting regional development in one coherent system represented by a dual graph. The edges of this graph can be naturally interpreted as regional growth barriers. This excludes, to a certain extent, the subjectivity resulting from a qualitative expert evaluation.

3. It takes into account spatial diversification of conditions for conducting business activity in individual communes. This can be perfectly exemplified by cooperation of the communes situated along the Elblag Canal. This inland water route is a course of infrastructural growth poles, taking advantage of natural factors in the form of a series of lakes situated in various communes. As results from Fig. 2, each of those communes have websites of the highest quality, which in the western part of the voivodship creates the turnpike of economic growth along the north-south axis.

4. The methodology used in the paper is based on the flow of economic information, both in individual communes and among them, which allows us to treat the entire voivodship as one system and to diagnose its strength 
and weaknesses.

5. The applied methodology is justified both theoretically and practically. The impact of technology on economic development has been a central issue in the theory of economics for long. However, in the latest years, a new economic school emerged, known as wikonomics, which is based on empirical patterns of economic cooperation. The graph theory combines both issues and makes it possible to introduce synergistic reasoning to economics.

6. The method facilitates real time monitoring of the economic growth in individual regions. The study can be replicated at any moment. Poles and turnpikes of economic growth are dynamic phenomena, as some of them can emerge and develop in economic spaces, while others can disappear. Comparison of maps in Fig. 2 and Fig. 3 allows us to examine the dynamics of those changes.

7. Dynamic studies make it possible to distinguish communes being de facto growth initiators, from those who are based only on imitation. This fact is of crucial importance for regional economic policy, as it helps to allocate resources and financial means in a more effective manner.

8. The applied method is not the last word on the subject of the regional growth pole identification and shows a potential for further improvement and development. Moving the discussion into the graph theory field provides the possibility for regional economics to enter new areas. This is beneficial for the development of interdisciplinary and transdisciplinary studies.

\section{Conclusions}

Basing the economic growth of the Warmian-Masurian voivodship on the historically conditioned infrastructural growth poles explains why it is one of the least-developed regions in Poland. Since 1945, few key investments, especially those related to innovation, have been implemented in the region. Two of the rare exceptions are the abovementioned fibre-optic network and the modernisation of the Elblacg Canal, completed in 2015, which cost PLN 115 million (EUR 27,515,911.37) [27]. It has to be highlighted that this structure has been serving its purpose without any major repairs for over 150 years [28]. The main source of wealth in those areas remains the transportation infrastructure erected by previous generations in previous centuries. There are plans for investments, e.g. development of the docks in Elblagg due to digging through the Vistula Spit, creating a medium-sized feeder port connecting the Tri-Cities and Russian ports, and further development of the ICT infrastructure as part of the 2025 Digital Plan for Warmia and Masuria.

A certain question related to the economic history of this region, though significant nowadays, comes to mind: Did Poland play any part in creating infrastructural growth poles in the region of Warmia and Masuria prior to 1945 ? It seems that the answer to this question is positive. Between 1226 and 1466, the region was ruled by the Teutonic Order. It contributed to the economic growth of those lands by replacing hand-mills with windmills. In 1466, as a result of the Second Peace of Thorn, the State of the Teutonic Order was divided into Royal Prussia and Teutonic Prussia (Ducal Prussia since 1525). In fact, both parts of Prussia became part of Poland, though the latter under fiefdom [29]. The golden period for windmills in the region of Warmia and Masuria was between the 15 th century and the 18th century (and later) when those lands were officially (or unofficially) part of the Polish economy. The location of windmills in the region from the turn of the 19th and 20th centuries is closely related to the present transportation infrastructure [30]. Windmills laid the path for the main trade and communication routes, which were essentially the same routes along which the fibre-optic network was recently constructed. It indicates that Poland substantially contributed to creating historically conditioned infrastructural growth poles in the region of Warmia and Masuria.

\section{References}

[1] R. Girejko, Methodology for Identification of Priorities and Growth Poles of Sustainable Regional Development in Belarus Based on Best International Practices, Technical Paper, March 13, 2015. Annex II. International technical support project: Support to Regional and Local Development in Belarus. This project is funded by the European Union. Project approved by the Council of Ministers of the Republic of Belarus, December 30, 2013, No. 1167. Registered by the Ministry of Economy, February 15, 2014, No. 2/14/000687.

[2] R. Fritsch, G. Fritsch, The Four-Color Theorem: History, Topological Foundations, and Idea of Proof, Springer-Verlag, New York 1998.

[3] R. Wilson, Four Colors Suffice: How the Map Problem Was Solved, Princeton University Press, Princeton 2014.

[4] K. Appel, W. Haken, Every Planar Map is Four Colorable, American Mathematical Society, Providence 1989.

[5] N. Robertson, D.P. Sanders, P. Seymour, R. Thomas, Electron. Res. Announce. Am. Math. Soc. 2, 17 (1996).

[6] N. Robertson, D. Sanders, P. Seymour, R. Thomas, J. Combinat. Theory Series B 70, 2 (1997).

[7] G. Gonthier, A Computer-Checked Proof of the Four Colour Theorem, Microsoft Research, Cambridge, retrieved March 15, 2017.

[8] G. Gonthier, Notic. Am. Math. Soc. 55, 1382 (2008).

[9] J.P. Steinberger, Trans. Am. Math. Soc. 362, 6633 (2010).

[10] S.G. Krantz, The Proof is in the Pudding: The Changing Nature of Mathematical Proof, Springer Science + Business Media, New York 2011.

[11] E.R. Swart, Am. Math. Monthly 87, 697 (1980).

[12] T. Tymoczko, J. Philos. 76, 57 (1979).

[13] D. Tapscott, A.D. Williams, Wikinomics: How Mass Collaboration Changes Everything, Portfolio, New York 2006. 
[14] A. Jakimowicz, D. Rzeczkowski, Acta Phys. Pol. A 129, 1011 (2016).

[15] S. Ahmed, Int. J. Comput. Informat. Technol. 3 130101 (2012), retrieved March 27, 2017.

[16] T. Bajerowski, M. Gerus-Gościewska, Zeszyty Naukowe Akademii Rolniczej im. Hugona Kottataja w Krakowie 353, 57 (1999) (in Polish).

[17] M. Gerus-Gościewska, T. Bajerowski, in: Theoretical Conceptions and Research Methods of SocioEconomic Geography and the Space Economy, Ed. H. Rogacki, Bogucki Wydawnictwo Naukowe, Poznań 2001, p. 283 (in Polish).

[18] P.C. Kainen, Quantum Interpretations of the Four Color Theorem, Conf. Proc., 2001, retrieved March 16, 2017.

[19] M.-C.O.S. Popescu, N.E. Mastorakis, Int. J. Appl. Math. Informat. 3, 17 (2009), retrieved March 16, 2017.

[20] R. Timofte, L. Van Gool, in: Advanced Topics in Computer Vision, Eds. G.M. Farinella, S. Battiato, R. Cipolla, Springer-Verlag, London 2013, p. 313.

[21] F. Perroux, Economics of the 20th Century, Presses Universitaires de France, Paris 1964 (in French).

[22] J. Grzeszczak, Growth Poles versus Forms of Polarised Space, Wydawnictwo Continuo, Wrocław 1999 (in Polish).
[23] Broadband Network in Eastern Poland (2017), retrieved May 28, 2017 (in Polish).

[24] P. Krzyżanowski, Completion of a Broadband Network in the Region of Warmia and Masuria, Komputer Swiat (2015), retrieved May 25, 2017 (in Polish).

[25] R. Dorfman, P.A. Samuelson, R.M. Solow, Linear Programming and Economic Analysis, McGrawHill, New York 1958.

[26] L.W. McKenzie, Econometrica 44, 841 (1976).

[27] The Revitalisation of the Elblag Canal, Regionalny Zarząd Gospodarki Wodnej w Gdańsku, Gdańsk 2015, retrieved May 12, 2017.

[28] R. Kowalski, Ostróda-Iława-Elblag. A History of Inland Sailing, Edytor-Wers, Olsztyn 2016 (in Polish).

[29] J. Małłek, Two Parts of Prussia: A Study of the History of Ducal Prussia and Royal Prussia in the 16th and 17th Centuries, Wydawnictwo Pojezierze, Olsztyn 1987 (in Polish), retrieved May 19, 2017.

[30] W. Chodkowska, M. Sabljak-Olędzka, Of Windmills in Warmia and Masuria and a Miller from Away..., Muzeum Budownictwa Ludowego - Park Etnograficzny w Olsztynku, Olsztynek 2016 (in Polish). 